\title{
Generating Quad Mesh of 3D Articulated Shape for Sculpting Modeling*
}

\author{
Jinliang $\mathrm{WU}^{* *}$ and Ligang LIU** \\ ** Department of Mathematics, Zhejiang University \\ Department of Mathematics, Zhejiang University, Hangzhou 310027, China \\ E-mail: jinliangwu@zju.edu.cn, ligangliu@zju.edu.cn
}

\begin{abstract}
In this paper, we propose a novel quad remeshing algorithm for articulated shapes. Different from existing methods that are essentially based on local analysis to align with local geometric features, our method focuses on the global structure and generates quad mesh with edge flow aligning with the skeleton. To achieve this, we firstly extract the skeleton from 3D shape. It captures the global structure of the shape and decomposes the shape into feature parts. We detect tube-like parts and build sweeping surface on them. For joint part connecting tube-like parts, we generate a smooth cross field interpolating the directions from its adjacent tube-like parts. Using this cross field, we generate the quad mesh of joint part. It smoothly connects adjacent sweep surfaces. The mesh models generated in our system greatly benefit the sculpting operators for sculpting modeling and skeleton-based animation.
\end{abstract}

Key words: Sculpting Modeling, Quadrangulation, Skeleton, Direction Field, Parametrization

\section{Introduction}

With the development of film and entertainment industry, faithful digital 3D models of high detailed objects are increasingly used. Many of them are created by the digital sculpting softwares. Although sculpting is still a very new method, it has become very popular approach for creating 3D character shapes with scan quality geometric detail in game and film industry in a few short years. It has been a new trend to 3D modeling. Many sculpting softwares are available, such as $\mathrm{ZBrush}^{(1)}$ and Mudbox ${ }^{(2)}$, Like multi-resolution modeling, the sculpting modeling considers a 3D modeling as a coarse-to-fine procedure. First, a coarse base mesh is built to represent the rough structure of the shape. The user then imports the base mesh into some sculpting package, subdivides it into higher resolution, and adds geometric and texture details ${ }^{(3)}$.

In the sculpting packages, quad meshes are usually preferred over triangle meshes. It is natural for subdivision. Quad mesh with good edge flow can improve the efficiency of mesh editing. Adding geometric details becomes easier. The good edge flow also has an important role in the animation, and allows you to control the deformation of the model conveniently. Lots of triangle meshes are available on the internet. Therefore, the conversion into quad meshes with good edge flow is an important requirement. The generated quad mesh can be used to provide a starting point for next sculpting modeling. It can save 3D modeling artists much time and effort.

Different from existing algorithms focusing primarily on producing quality quad mesh of good alignment with local geometric features, We put more emphasis on global alignment with the structural features of the processed model in the remeshing. We extract the skeleton from 3D shape. It abstracts the geometry and topology of 3D shapes and 
decomposes the model into several feature parts. We construct the corresponding quad mesh on each feature part by its structure. Finally, all generated quad meshes are connected together seamlessly to get the resulting quad mesh. It globally aligns with the extracted skeleton.

For a quad mesh, its topology plays an important part in modelling and animation. A nice topology will make it possible to build a model with less geometry but still look accurate and realistic. And a bad topology will also affect the animation deformer. Our algorithm allows the user to edit and refine the quad mesh topology during the remeshing.

The paper is organized as follows. An overview of related literature is given in $\S 2$. Section 3 addresses tube-like quadrangulation. The construction of quad mesh on joint part is covered in $\S 4$. In $\S 5$, Implementation details are explained. Some experimental results are presented in $\S 6$ and conclusions follow this.

\section{Related Work}

\subsection{Skeleton Extraction}

Curve skeletons are 1D structures that are often used to represent a simplified version of the geometry and topology of a 3D object geometry ${ }^{(4)}$. It is effective shape abstraction that have been used in various applications including shape matching ${ }^{(5)}$, animation ${ }^{(6)}$, and mesh segmentation ${ }^{(7)(8)}$. In addition to curve skeletons, the medial axis ${ }^{(9)}$ is another notable means for obtaining a topological representation of a shape, which provides a powerful dual representation of the shape surface. However, it contains surface elements and is difficult to model and manipulate. The work of Dey and Sun ${ }^{(10)}$ employs Voronoi diagrams and extracts a curve skeleton by pruning the extracted approximation of the medial axis. However, it is sensitive to sampling conditions and noise. The work of Patane ${ }^{(11)}$ extracts and links critical points of a function over the mesh surface via Reeb graphs. Sharf et al. ${ }^{(12)}$ extract skeletons both from point clouds and polygonal meshes by applying a deformable model evolution. The recent work of $\mathrm{Au}$ et al. ${ }^{(8)}$ skeletonizes a shape by shrinking it using constrained Laplacian smoothing. Excellent results are obtained. Miklos et al. ${ }^{(13)(14)}$ introduce a family of skeletons that captures the important features of a shape in a scale-adaptive way.

\subsection{Sweeping Modeling}

A sweep surface is defined as the envelope formed by the transformation of a cross section curve along a second curve ${ }^{(15)}$. Generally, sweeps of 2D cross sections are known as generalized cylinders. Sweeping surfaces are widely used in many geometric modeling applications. Now, Most CAD commercial packages allow generation of volumes by extruding a surface along an axis. Sweeping surface generation methods are intensively explored $^{(16)-(18)}$. Tai and Loe ${ }^{(19)}$ introduce sinusoidal blending functions and deformation function to deform uniform periodically swept surfaces. Hyun et al. ${ }^{(20)}$ and Karla et al. ${ }^{(21)}$ deform a virtual character's arms and legs using sweep surfaces. Hyun et al. ${ }^{(22)}$ use sweep surfaces as a control mechanism to deform polygonal meshes of human models. Yoon et al. ${ }^{(23)}$ extend this work to alter the shape of an object by deforming the sweep surface of the object.

\subsection{Quad Remeshing}

A large variety of approaches have been brought forth in quad remeshing to fulfill the ever increasing need of quad representations. An intensive survey on quad remeshing is beyond the scope of this paper. For a comprehensive overview, the reader is referred to Ref. (24) and the references cited therein. The work of Alliez et al. ${ }^{(25)}$ integrates principal curvature lines in the parameter domain and generates a quad-dominant output mesh by intersections of these lines. They are extended by Marinov and Kobbelt ${ }^{(26)}$ to arbitrary meshes by applying curvature line integration on the underlying surface. Dong et al. ${ }^{(27)}$ present an algorithm which traces isolines in two conjugate harmonic vector fields. However, it needs necessary user intervention and suffers the loss of feature alignment to generate more regular results. More recent parametrization based techniques have also proven to be a powerful tool for quad remeshing. Ray et al. ${ }^{(28)}$ parameterize surfaces of arbitrary topology by defining two piecewise linear periodic functions guided by two 


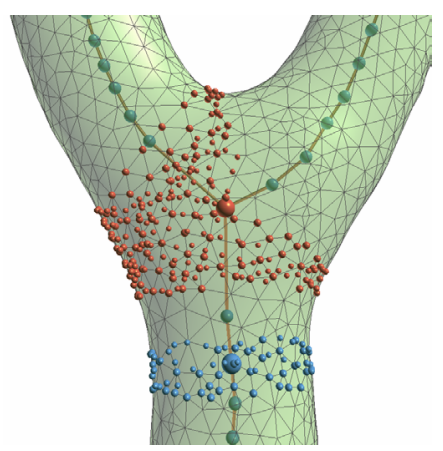

Fig. 1 An illustration of skeleton-mesh mapping. Each skeleton node records vertices on the original mesh collapsed to it.

orthogonal input vector fields defined on the surface. The quadrilaterals are extracted from the bivariate parameterization function. Kälberer et al. ${ }^{(29)}$ develop a linear algorithm by converting a given frame field into a single vector field on a branched covering and produce quadrilateral meshes with fewer singularities. Marinov and Kobbelt ${ }^{(30)}$ propose a two-stage remeshing algorithm that firstly segments the input mesh into patches using a slight variant of variational shape approximation and then generates the output mesh by computing an optimized quad mesh for every patch. Dong et al. ${ }^{(31)}$ obtain a segmentation from the MorseSmale complex of the eigenfunctions of the Laplacian. It generates high quality remeshing with few extraordinary vertices. However, features are usually not well captured. Their method is extended by Huang et al. ${ }^{(32)}$ to add alignment and orientation control to the parametrization. However, high quality results is still involved and requires an experienced user.

\section{Tube-Like Parts Quadrangulation}

\subsection{Skeleton Extraction}

The skeleton of a shape, especially an articulated shape, provides an intuitive and effective abstraction. Au et al. ${ }^{(8)}$ propose a method of extracting a $1 \mathrm{D}$ curve-skeleton by carefully shrinking a shape using constrained Laplacian smoothing. A curve-skeleton $\mathbf{S}=(\mathbf{U}, \mathbf{B})$ with skeleton nodes $\mathbf{U}$ and edges $\mathbf{B}$, where $\mathbf{U}=\left[\mathbf{u}_{1}^{T}, \mathbf{u}_{2}^{T}, \ldots, \mathbf{u}_{m}^{T}\right]^{T}$ are the node positions. The skeletal nodes are classified into three types ${ }^{(33)}$ : end node (if it connects to only one edge), connection node (if it connects to two edges), and joint node (if it connects to more than two edges). We decompose the skeletal curve-skeleton structure into several limbs, each of which has no joint nodes.

The skeleton extraction method ${ }^{(8)}$ gives rise to a skeleton-mesh mapping. For each skeleton node $k$, the skeleton-mesh mapping records the set of vertices $\Pi_{k}$ on the original mesh that are contracted and collapsed to it. For non-joint nodes, its corresponding vertices form a cylinder-like shape. For joint nodes, its corresponding vertices form a sphere-like shape $^{(8)}$. Figure 1 shows an example of the induced skeleton-mesh mapping. It gives a fine correspondence between the curve-skeleton and the original mesh which is useful for our next tubular detection.

\subsection{Tube-Like Parts Detection}

We utilize the extracted skeleton and skeleton-mesh mapping to detect parts of the shape that can be locally approximated by tubular sections. For each skeleton node $k$, we firstly find the optimal cutting plane $\pi_{k}$ through $\mathbf{u}_{k}^{T}$ whose normal direction $\mathbf{v}_{k}$ is orthogonal to the normals associated with the set of vertices $\Pi_{k}$ in a least squares sense. We find the normal direction $\mathbf{v}_{k}$ by solving the optimization:

$$
\underset{\left\|\mathbf{v}_{k}\right\|=1}{\arg \min } \sum_{\mathbf{p}_{i} \in \Pi_{k}}\left(\mathbf{n}_{i} \mathbf{v}_{k}^{T}\right)^{2},
$$




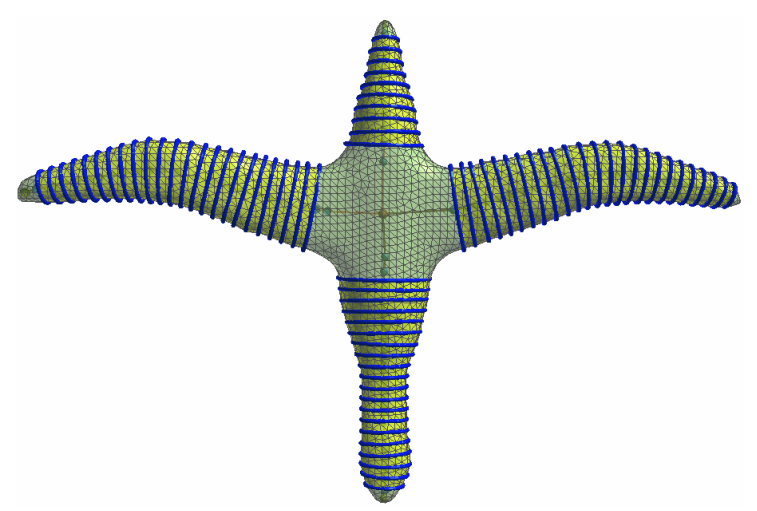

Fig. 2 The detected tube-like parts. The blue ellipse is used in detection.

where $\mathbf{p}_{i}$ is the mesh vertex and $\mathbf{n}_{i}$ is its corresponding normal. This problem can be reduced to find the smallest eigenvector of the covariance matrix ${ }^{(34)} \sum_{\mathbf{p}_{i \in \Pi_{k}}}\left(\mathbf{n}_{i} \mathbf{n}_{i}^{T}\right)^{2}$.

For each skeleton node $k$, we find its optimal cutting plane and refer it to cross-sectional plane. We identify the subset $\mathbf{N}$ of corresponding vertices $\Pi_{k}$ that is near to its cross-sectional plane. The vertices in the subset $\mathbf{N}$ are projected to the cross-sectional plane. We solve an optimal ellipse fitting these projected vertices.

We set the skeleton node with the smallest fitting error as seed node. From the seed node, we search reliable tubular part in both directions on its belonging limb. A verification procedure controls the reliable tubular part expansion. The stop criterion is given by the abrupt variation of the size of fitting ellipse and the direction of cross-sectional plane.

\subsection{Sweep Surface of A Moving Ellipse}

For tube-like part (Fig. 2), sweep surface can generate the corresponding quad mesh easily. We represent a sweep surface (Fig. 3) by generating cross section curve $\mathbf{G}$ along a skeleton line ${ }^{(34)} \mathbf{C}$. The cross section $\mathbf{G}_{k}$ at each skeleton node $k$ is represented as a planar (closed) polygon with $m$ points $\mathbf{G}_{k}=\left\{\mathbf{G}_{k}^{1}, \mathbf{G}_{k}^{2}, \ldots, \mathbf{G}_{k}^{m}\right\}$ sampling from the fitting ellipse. We arbitrarily assign a vector in the plane containing $\mathbf{G}_{k}$ as the local $x$-axis at $\mathbf{C}_{1}$, and then propagate the local coordinate frame to other cross section curve $\mathbf{G}_{k}$ along the skeleton line $\mathbf{C}$ using a rotation minimizing formulation ${ }^{(35)}$.

The sweep surface is builded on the underlying tubular part surface. It consists of simple quad faces. We refine the generated sweep surface to tightly fit the input shape $\mathbf{M}$. We project its vertex to the input shape along the vector going from the center of its corresponding ellipse to it. This ensures that the projected vertices are still on the

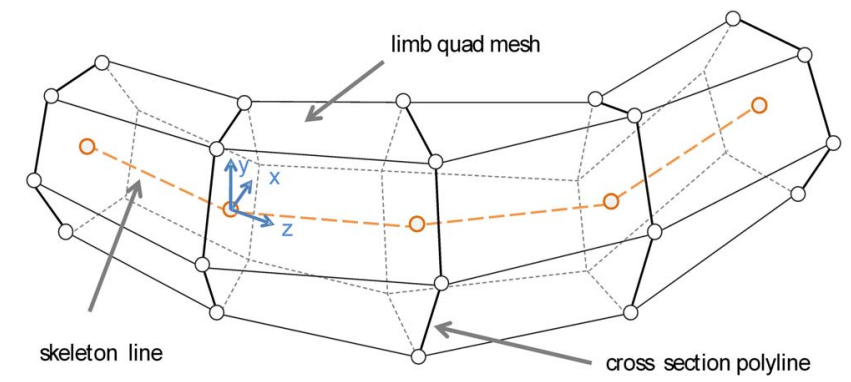

Fig. 3 We generate a limb quad mesh by sweeping cross section polyline along a skeletal line. Cross section polyline is sampled from fitting ellipse. 


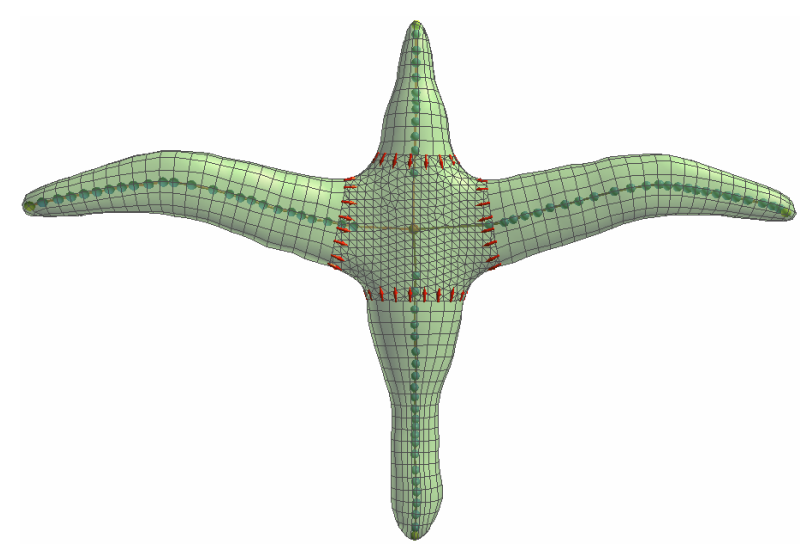

Fig. 4 Limb quad mesh builded on tube-like part. It also provides initial directions for the generation of cross field.

cross-sectional plane. We refer every generated sweep surface to limb quad mesh (Fig. 3). According to tube-like part, the input model $\mathbf{M}$ is now classified into three types: tube-like part, tip part (if it connects to only one tube-like part), joint part (if it connects to more than two tube-like parts).

\section{Limb Quad Mesh Connection}

Limb quad mesh has good edge flow which is the same with the skeleton and aligns with the global structure of the input shape. Next, we build a quad mesh on joint part providing a smooth transition between the edge flow of its adjacent limb quad meshes.

Different from tube-like part, the joint part is generally non-cylindrical and lacks a clear direction. We use a diffusion operator based on a cross field formulation to globally and smoothly propagate the directions of limb quad mesh to the joint part (Fig. 4). Guided by the consistent cross field, we quadrangulate the joint part. The generated quad mesh on joint part connects adjacent limb quad mesh smoothly and provides a smooth transition between the edge flow.

\subsection{Interpolating Cross Field Generation}

A cross field (Fig. 5(a)), represented on each triangle $T$, is a set of four vectors. It is

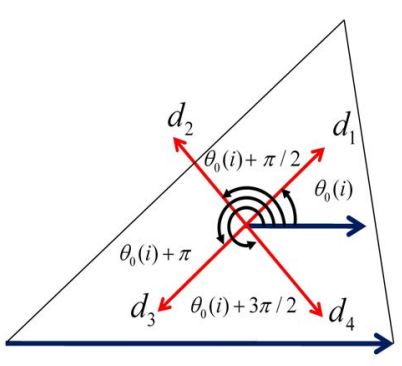

(a)

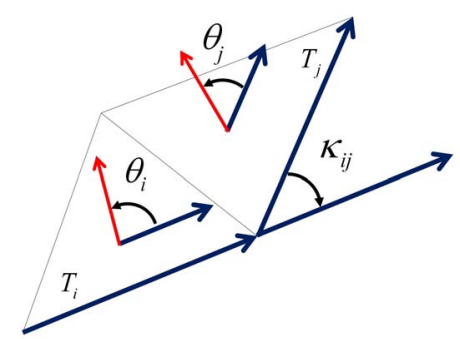

(b)

Fig. 5 The cross field. (a) The cross field on a triangle is a set of vectors $\mathbf{d}_{k}, k \in\{1,2,3,4\}$ defined as the images of a reference vector $\mathbf{r}_{i}$ by rotations of $\theta_{0}(i)+k \pi / 2$;

(b) Matching between cross fields on adjacent triangles. 


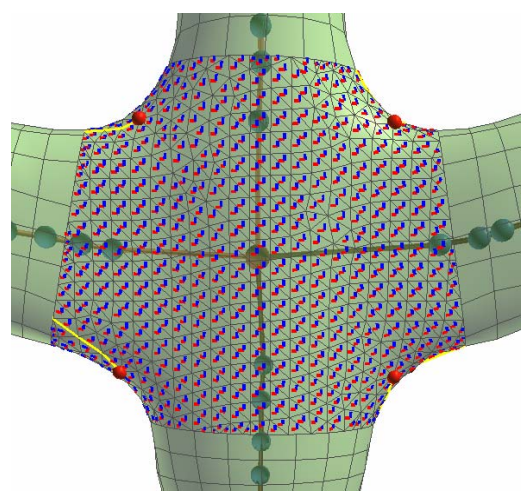

Fig. 6 The smooth cross field generated on joint part. Singularities are detected.

defined as the images of a reference vector $\mathbf{r}_{i}$ by rotations of $\theta_{0}(i)+k \pi / 2^{(36)}$. Each edge is assigned an integer called period-jump.

Let $T_{i}$ and $T_{j}$ be two adjacent triangles, $d\left(T_{i}\right)$ and $d\left(T_{j}\right)$ are the direction field on triangle $T_{i}$ and $T_{j}$. The discrete curvature of a direction field can be defined as the oriented angle that brings $d\left(T_{i}\right)$ to $d\left(T_{j}\right)$ in a common coordinate frame:

$$
C\left(\theta_{i}, \theta_{j}\right)=\theta_{i}+\kappa_{i j}+\frac{\pi}{2} p_{i j}-\theta_{j}
$$

where: $\kappa_{i j} \in(-\pi, \pi]$ is the angle of a rotation between local frames and $p_{i j}$ is an integer valued period jump across edge $e_{i j}$ used to solve the ambiguity in the interpolation from $d\left(T_{i}\right)$ to $d\left(T_{j}\right)$ (Fig. 5(b)).

The singularity index at a vertex $v$ is defined as ${ }^{(36)}$ :

$$
I\left(v_{i}\right)=\frac{1}{2 \pi}\left(\sum_{e_{i j} \in N\left(v_{i}\right)} \kappa_{i j}+K_{i}\right)+\sum_{e_{i j} \in N\left(v_{i}\right)} \frac{p_{i j}}{4}
$$

where $K_{i}$ is the angle defect defined to be $2 \pi$ minus the sum of the angles at the corners of the triangles at vertex $v_{i}$. Indices are multiples of $1 / 4$ and only singularities of the cross field have a nonzero value, e.g. $1 / 4$ and (-1/4). Note that the indices of singularities depend only on the $p_{i j}$, whatever the choice for $\theta$.

Singularities have a great impact on the global geometric structure. Lots of singularities will destroy good edge flow. We aim to capture the global shape of the object with the minimal number of singularities.

Motivated by the work of Ray et al. ${ }^{(37)}$, we reduce the number of singularities for high detailed objects by smoothing the density of curvature. We use the Gaussian smoothing algorithm ${ }^{(37)}$ to smooth the angle defect $K_{i}$ :

$$
K_{j}^{c o r r}=\sum_{v_{i}} \frac{c_{i j} K_{i}}{\sum_{v_{k}} c_{i k}}, \quad c_{i j}=e^{\left(-\frac{D_{i j}}{\sigma}\right)^{2}}
$$

where $\mathrm{D}_{i j}$ is the distance from vertex $v_{i}$ to vertex $v_{j}$ computed by a Dijkstra algorithm. $\sigma$ is a Gaussian radius controlling the extent of smoothing. Then the new target curvature $C^{t}\left(\theta_{i}, \theta_{j}\right)$ is calculated for each edge $e_{i j}$ to compensate for the difference of angle defect: $\sum_{e_{i j} \in N\left(v_{i}\right)} C^{t}\left(\theta_{i}, \theta_{j}\right)=K_{i}^{c o r r}-K_{i}$ with minimal norm.

For joint part, the directions on border triangles are fixed by the growth direction of limb quad mesh. We find a smooth, interpolating cross field by minimizing the new smoothness energy:

$$
E_{\text {smooth }}=\sum_{e_{i j} \in E}\left(C\left(\theta_{i}, \theta_{j}\right)-C^{t}\left(\theta_{i}, \theta_{j}\right)\right)^{2} .
$$

In this smoothing process, only important geometric features are kept and the number of singularities are reduced. The only tuning parameter is the Gaussian radius $\sigma$. 


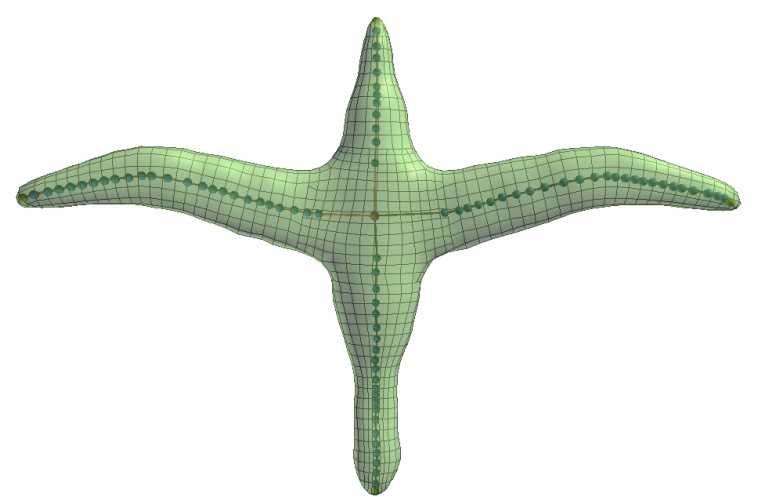

Fig. 7 The generated quad mesh. It aligns with the global structure of the processed model.

\subsection{Joint Quad Mesh Generation}

Using this cross field, we generate the quad mesh of joint part. We parameterize the joint part by fitting the parametrization gradient to the smooth cross field. To guarantee a pure quadrangulation, we utilize the greedy mixed-integer solver method ${ }^{(38)}$.

We briefly review the algorithm and the main steps are:

(1) Cut open the mesh and obtain a patch that is topologically equivalent to a disk. Detect singularities and add paths connecting each singularity to the cut graph (see Refs. (12) and (18) for more details).

(2) Propagate a globally consistent orientation in the cross field and establish a zero-rotation across all inner edges.

(3) Matching across seams. Constrain $u$ and $v$ directions on the cut mesh globally consistent. For a cut edge $e=\overline{p q}$, with parametric positions $\mathrm{p}=\left(\mathrm{u}_{1}, \mathrm{v}_{1}\right)$ and $\mathrm{q}=\left(\mathrm{u}_{2}, \mathrm{v}_{2}\right)$ on one side of the cut, and $p^{\prime}=\left(u_{1}^{\prime}, v_{1}^{\prime}\right)$ and $q^{\prime}=\left(u_{2}^{\prime}, v_{2}^{\prime}\right)$ on the other side. They are related by

$$
p^{\prime}=R_{e_{i}} p+t_{e} \quad q^{\prime}=R_{e_{i}} q+t_{e}
$$

where $\mathrm{R}_{e_{i}}$ is the rotation between the orientations in neighboring faces, and $t_{e}$ is unknown integer variables. $\mathrm{T}$ :

(4) Minimize the fit energy defined as the integral of orientation energy on each triangle

$$
\sum_{T \in \mathbf{M}} \operatorname{area}(T)\left(\left\|h \nabla u-\mathbf{u}_{T}\right\|+\left\|h \nabla v-\mathbf{v}_{T}\right\|\right)
$$

for parameterization $u$ and $v$. The scale factor $h$ controls the edge length of the resulting quad mesh. The vectors $\mathbf{u}_{T}$ and $\mathbf{v}_{T}$ correspond to the cross field directions $\theta_{i}$ and $\theta_{i}+\pi / 2$ in $T$.

All singularities are set in integer positions to guarantee a pure quadrangulation. Once the parameterization is computed, we generate a quad mesh by tracing the integer parametric lines on the surface. It might happen that the orientation of a mapped triangle flips in the algorithm described above. To solve this problem, the last step may be repeated several times with adapted triangle weights added into the energy formulation to penalize high local parametric distortions.

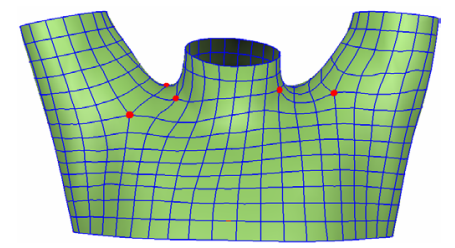

(a)

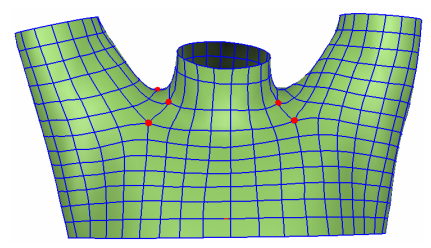

(b)

Fig. 8 Singularity adjustment. (a) The quad mesh without singularity adjustment does not respect local symmetry. (b) The quad mesh with singularity adjustment. 


\section{Implementation Details}

\subsection{Adjust Singularity}

The field topology (singularities) indirectly determines the structure of quad mesh. The singularity in the cross field causes a valence 3 and 5 vertex in the generated quad mesh. Their positions capture the structure of the quad mesh and affects its edge flow. A quadrilateral mesh with singularities placed at meaningful positions is of great interest, since it greatly benefits for further sculpting modeling and animation.

To avoid the heavy computational cost of singularity relocation in Ref. (18) and allow the user to have an direct control over the result. After the smooth field is built, if singularities are not at appropriate positions, for instance not to respect local symmetries. The user can shift singularities to semantically meaningful positions to improve the quality of generated quad mesh (Fig. 8).

For the new smoothness energy, it is easy to move singularities. We only need to modify the corresponding smooth curvature values $K_{i}^{\text {corr }}$ when moving a singularity from one place to another ${ }^{(37)}$. Moving an index $I$ singularity from $v_{0}$ to $v_{1}$ is handled by adding $\frac{\pi}{2} I$ to $K_{v_{1}}^{\text {corr }}$ and subtracting $\frac{\pi}{2} I$ to $K_{v_{0}}^{\text {corr }}$.

\subsection{Stitch Mesh}

It is easy to stitch limb quad mesh and joint quad mesh. The corresponding border vertices are on the same cross-sectional plane. We compute the center of border vertices and arbitrarily assign a vector in the cross-sectional plane as the local $x$-axis. The polar coordinate of every vertex is computed. For every vertex on the border of limb quad mesh, we find its nearest vertex on the border of joint quad mesh by angular coordinate and merge them. For other vertices, we interpolate their positions by neighbor vertices.

\subsection{Quad Mesh Template}

In our implementation, we sample $m=4 * n$ vertices on the fitting ellipse according to the target edge length of quad mesh. For tip part with a small number of triangles, we provide a simple approach for quadrangulation. A quad mesh template is used for these tip parts (Fig. 9(a)). We firstly close the end of the limb quad mesh connecting to this tip part using quad mesh template. Then every vertex in the template finds its corresponding position on tip part. For every vertex of the template, we shoot a line along the normal of cross-sectional plane and find the intersection with this tip part as its new position (Fig. $9(b))$.

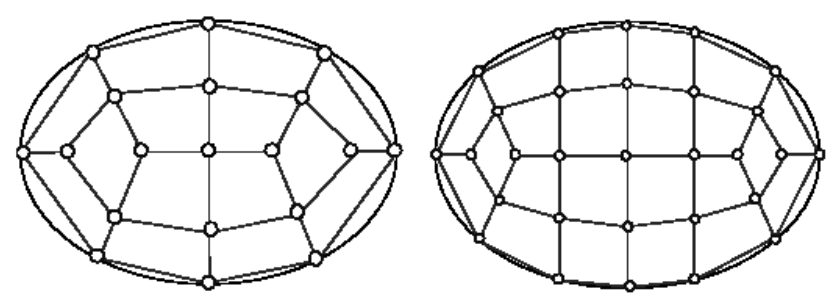

(a)
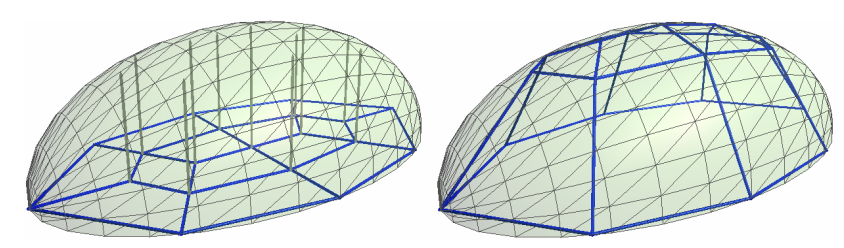

(b)

Fig. 9 The quad mesh template. (a) The template used at the end of limb quad mesh.

(b) The templates are projected to the original mesh. 


\section{Experimental Results}

In this section, we show more results of our method and discuss some of its properties. We evaluate the results of our algorithm in several ways. The main criterion is that the output quad mesh aligns with the global structure of the processed model. We then discuss limitations of our method.

For 3D articulated shapes, they have simple and intuitive skeleton. The skeleton divides the model into several feature parts. These parts always have clear direction and response to the global structure of the model. Treating these parts separately and then connecting corresponding results smoothly is a correct method.

Figure 10 shows some remeshing results using our method. Note that all the results have good edge flow which aligns with the global structure of the model. For the horse model in Fig. 10(b), we firstly detect its neck part, waist part, leg part and tail part and build corresponding sweep surface. These main parts determine the structure of quad mesh and the singularities locate in semantically meaningful positions. Our system can handle models of ring structure (Fig. 10(c)). For some industrial models, we can also get good results. For the airplane in Fig. 10(d), its airfoil and fuselage parts are firstly detected and the global structure of generated quad mesh is good.

All our experiments are carried out on a commodity PC with Intel Core2Duo $2.53 \mathrm{GHz}$ processor and 2 GB RAM. The tube-like part detection takes 1-3s, the smooth cross field generation 20-30s, the joint quad mesh generation 20-30s. The total time is around 1 minutes.

On the implementation level, there is still some room for improvement. Our method focuses on generating quad mesh models which benefit the sculpting operators for sculpting modeling and skeleton-based animation. It is not suitable for architectures and mechanical objects with sharp features.

\section{Conclusions}

We have presented a novel approach for turning an unstructured triangle mesh into a quad mesh which globally aligns with the shape of the model. Our system focuses on articulated shapes. The output quad mesh has good edge flow globally aligning with the skeleton of the processed model and singularities emerging only for significant features. It greatly benefits the sculpting operators for sculpting modeling and can be directly used in many other 3D softwares. It provides a good starting point in the design of a model.

One key component of our method is that we get the global structure of one shape by its skeleton. For articulated shapes with generally tubular components, skeleton provides an intuitive and powerful abstraction. We exploit the skeleton to detect tube-like parts and guide the quadrangulation of the whole shape.

Another component is that we reduce the number of singularities in the generated quad mesh by smoothing the density of curvature. The users are allowed to edit the field topology to determine the position of singularities in the generated quad mesh.

\section{Acknowledgments}

We would like to thank the anonymous reviewers for their valuable comments and suggestions. This work is supported by the National Natural Science Foundation of China (61070071), the 973 National Key Basic Research Foundation of China (2009CB320801), and the Fundamental Research Funds for the Central Universities (2010QNA3039). 


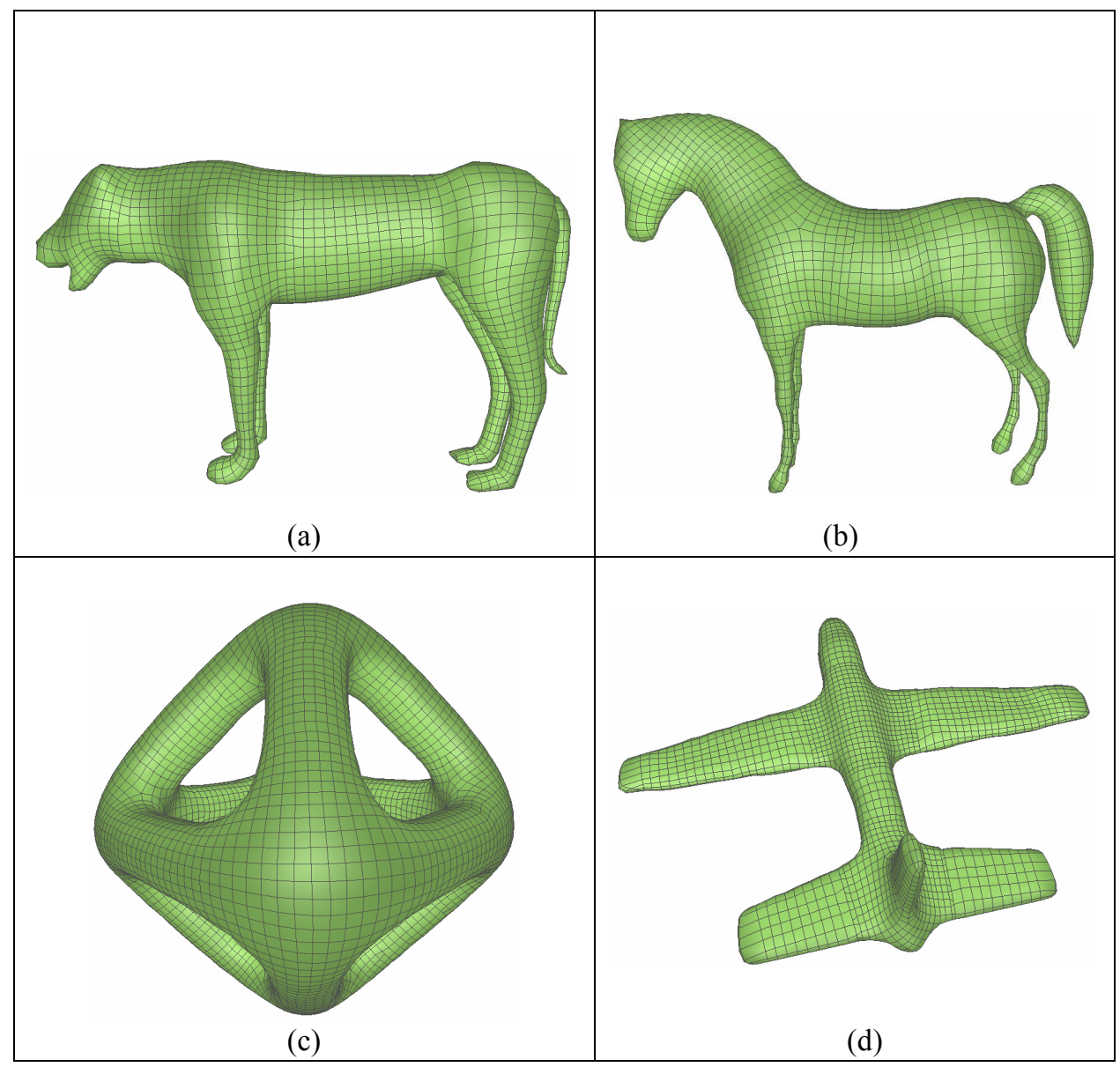

Fig. 10 Some remeshing results generated by our method.

\section{References}

(1) Pixologic, Zbrush. (online), available from $<$ http://www.zbrush.com $>$, (accessed 2011).

(2) Autodesk, Mudbox. (online). available: from http://usa.autodesk.com/adsk/servlet/pc/index?id=13565063\&siteID=123112>, (accessed 2011).

(3) Ji, Z., Liu, L. and Wang, Y., B-Mesh: A Modeling System for Base Meshes of 3D Articulated Shapes, Computer Graphics Forum, Vol. 29, No.7(2010), pp.2169-2178.

(4) Cornea, N. D., Silver, D. and Min, P., Curve-skeleton properties, applications, and algorithms, IEEE Transactions on Visualization and Computer Graphics, Vol. 13, No.3 (2007), pp.530-548.

(5) Hilaga, M., Shinagawa, Y., Kohmura, T. and Kunii, T. L., Topology matching for fully automatic similarity estimation of 3D shapes, Proceedings of the 28th annual conference on Computer graphics and interactive techniques, (2001), pp.203-212.

(6) Lewis, J. P., Cordner, M. and Fong, N., Pose space deformation: a unified approach to shape interpolation and skeleton-driven deformation, Proceedings of the 27th annual conference on Computer graphics and interactive techniques, (2000), pp.165-172. 
(7) Li, X., Woon, T., Tan, T. and Huang, Z. Decomposing polygon meshes for interactive applications, Proceedings of the 2001 symposium on Interactive $3 D$ graphics, (2001), pp.35-42.

(8) Au, O. K.-C., Tai, C.-L., Chu, H.-K., Cohen-Or, D. and Lee, T.-Y., Skeleton extraction by mesh contraction, ACM Transactions on Graphics, Vol.27, No.3(2008), pp. 44-52.

(9) Siddiqi, K. and Pizer, S., Medial Representations: Mathematics, Algorithms and Applications, (2008), Springer.

(10) Dey, T. K. and Sun, J., Defining and computing curve-skeletons with medial geodesic function, Proceedings of the fourth Eurographics symposium on Geometry processing, (2006), pp.143-152.

(11) Patane, G., Spagnuolo, M. and Falcidieno, B., Reeb graph computation based on a minimal contouring, Proceedings of IEEE International Conference on Shape Modeling and Applications, (2008), pp.73-82.

(12) Sharf, A., Lewiner, T., Shamir, A. and Kobbelt, L., On-the-fly curve-skeleton computation for 3D shapes, Computer Graphics Forum, Vol.26, No.3(2007), pp. 323-328.

(13) Giesen, J., Miklos, B., Pauly, M. and Wormser, C., The scale axis transform, Proceedings of the 25th annual symposium on Computational geometry, (2009), pp. 106-115.

(14) Miklos, B., Giesen, J. and Pauly, M., Discrete scale axis representations for 3D geometry, ACM Transactions on Graphics, Vol. 29, No.4(2010), pp.1-10.

(15) Elber, G., Global error bounds and amelioration of sweep surfaces, Computer-Aided Design, Vol. 29, No.6(1997), pp. 444-447.

(16) Bloomenthal, M. and Riesenfeld, R. F., Approximation of sweep surfaces by tensor product NURBS, Proceedings of Curves and Surfaces in Computer Vision and Graphics II, (1991), pp.132-154.

(17) Schmidt, R. and Wyvill, B., Generalized sweep templates for implicit modeling, Proceedings of the 3rd international conference on Computer graphics and interactive techniques in Australasia and South East Asia, (2005), pp.187-196.

(18) Seong, J.-K., Elber, G. and Kim, M.-S., Trimming local and global self-intersections in offset curves/surfaces using distance maps, Computer-Aided Design, Vol. 38, No.3(2006), pp.183-193.

(19) Tai, C. L. and Loe, K. F., Surface design via deformation of periodically swept surfaces, The Visual Computer, Vol.12, No.10 (1996), pp.475-483.

(20) Hyun, D. E., Yoon, S. H., Kim, M. S. and Juttler, B., Modeling and deformation of arms and legs based on ellipsoidal sweeping, Proceedings of the 11th Pacific Conference on Computer Graphics and Applications, (2003), pp. 204-212.

(21) Kalra, P., Magnenat-Thalmann, N., Moccozet, L., Sannier, G., Aubel, A. and Thalmann, D., Real-time animation of realistic virtual humans, IEEE Computer Graphics and Applications, Vol.18, No.5 (1998), pp.42-56.

(22) Hyun, D. E., Yoon, S. H., Chang, J. W., Seong, J. K., Kim, M. S. and Jüttler, B., Sweep-based human deformation, The Visual Computer, Vol.21, No.8-10(2005), pp. 542-550.

(23) Yoon, S. H. and Kim, M. S., Sweep-based freeform deformations, Computer Graphics Forum, Vol. 25, No. 3(2006), pp.487-496.

(24) Alliez, P., Ucelli, G., Gotsman, C. and Attene, M., Recent advances in remeshing of surfaces, Research report, AIM@SHAPE Network of Excellence, (2005).

(25) Alliez, P., Cohen-Steiner, D., Devillers, O., Levy, B. and Desbrun, M., Anisotropic polygonal remeshing, ACM Transactions on Graphics, Vol.22, No.3(2003), pp. 485-493. 
(26) Marinov, M. and Kobbelt, L., Direct anisotropic quad-dominant remeshing, Proceedings of the 12th Pacific Conference on Computer Graphics and Applications, (2004), pp.207-216.

(27) Dong, S., Kircher, S. and Garland, M., Harmonic functions for quadrilateral remeshing of arbitrary manifolds, Computer-Aided Geometric Design, Vol.22, No.3(2005), pp.392-423.

(28) Ray, N., Li, W. C., Lévy, B., Sheffer, A. and Alliez, P., Periodic global parameterization, ACM Transactions on Graphics, Vol.25, No.4(2006), pp. 1460-1485.

(29) Kälberer, F., Nieser, M. and Polthier, K., Quadcover - surface parameterization using branched coverings, Computer Graphics Forum, Vol.26, No.3(2007), pp. 375-384.

(30) Marinov, M. and Kobbelt, L., A robust two-step procedure for quad-dominant remeshing, Computer Graphics Forum, Vol.25, No.3(2006), pp. 537-546.

(31) Dong, S., Bremer, P.-t., Garland, M., Pascucci, V. and Hart, J. C., Spectral surface quadrangulation, ACM Transactions on Graphics, Vol.25, No.3(2006), pp.1057-1066.

(32) Huang, J., Zhang, M., Ma, J., Liu, X., Kobbelt, L. and Bao, H., Spectral quadrangulation with orientation and alignment control, ACM Transactions on Graphics, Vol. 27, No.5(2008), pp. 1-9.

(33) Wu, F.-C., Ma, W.-C., Liang, R.-H., Chen, B.-Y. and Ouhyoung, M., Domain connected graph: the skeleton of a closed 3D shape for animation, The Visual Computer, Vol. 22, No.5(2006), pp.117-135.

(34) Li, G., Liu, L., Zheng, H. and Mitra, N. J., Analysis, reconstruction and manipulation using arterial snakes, ACM Transactions on Graphics, Vol. 29, No.6(2010), pp. $1-10$.

(35) Wang, W., Jüttler, B., Zheng, D. and Liu, Y., Computation of rotation minimizing frames, ACM Transactions on Graphics, Vol. 27, No.1(2008), pp.1-18.

(36) Ray, N., Vallet, B., Li, W. C. and Lévy, B., N-symmetry direction field design, ACM Transactions on Graphics, Vol. 27, No.2(2008), pp. 1-13.

(37) Ray, N., Vallet, B., Alonso, L. and Lévy, B., Geometry-Aware Direction Field Processing, ACM Transactions on Graphics, Vol.29, No.2(2009), pp.1-11.

(38) Bommes, D., Zimmer, H. and Kobbelt, L., Mixed-integer quadrangulation, ACM Transactions on Graphics, Vol. 28, No.3(2009), pp. 1-10. 